\title{
HALLAZGO DE ADESMIA BIJUGA PHIL. (FABACEAE) EN LA ZONA COSTERA, REGION DEL MAULE, CHILE CENTRAL
}

\author{
REDISCOVERY OF ADESMIABIJUGA PHIL. (FABACEAE) IN THE COASTAL \\ ZONE, MAULE REGION, CENTRAL CHILE
}

\author{
Steffen Hahn \& Persy Gómez \\ Jardín Botánico de la Universidad de Talca, Casilla 747, Talca, Chile \\ shahn@utalca.cl
}

\section{ABSTRACT}

This article reports the rediscovery of the specie Adesmia bijuga Phil. (Fabaceae), in the coastal zone of the Maule

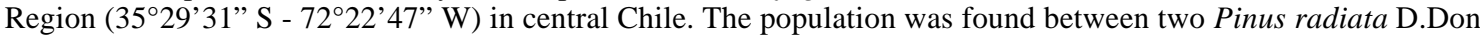
plantations and species of the sclerophyllous shrub. Its state of conservation is unknown.

El género sudamericano Adesmia de la familia Fabaceae, con más de 230 especies, presenta una amplia distribución en las regiones áridas de Chile, Argentina, Bolivia, Perú, sur de Brasil y Uruguay (Burkart 1967). Para Chile se citan aproximadamente 140 especies nativas (Marticorena \& Quezada 1985).

En este artículo se informa del hallazgo de Adesmia bijuga Phil., descrita por R. A. Philippi (1884), sobre una muestra encontrada por Raphael Azocart en la zona de Constitución, Región del Maule. A partir de esa fecha la especie no fue observada ni recolectada, sino sólo hasta el año 2003. En una excursión, el primer autor de este trabajo recolectó material en la Cordillera de la Costa de la Región del Maule (Fig. 1). Posteriormente en el año 2006 y en la misma localidad, nuevamente se recolecta material vegetal y otros antecedentes del ambiente del hallazgo. La confirmación taxonómica de la especie se basó en las descripciones de Philippi (1884), Reiche (1897) y consulta al herbario del Museo Nacional de Historia Natural (SGO), Santiago.

El género Adesmia presenta 2 subgéneros; Adesmia y Acanthadesmia con 35 y 10 series respectivamente. La serie Loudoniae del subgénero Adesmia presenta solamente 3 especies: Adesmia bijuga Phil., A. loudonia Hook. \& Arn y A. pirionii I.M.Johnst. (Burkart 1967).
La población de $A$. bijuga se encontró en el fundo San Pedro, Las Cañas, comuna de Constitución de la cordillera costera de la Región

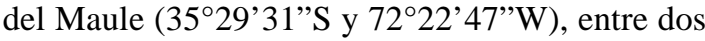
plantaciones, una juvenil y una adulta de la especie Pinus radiata D.Don y especies del matorral esclerófilo, entre las cuales destacan; Baccharis rhomboidalis J.Remy subsp. truncata (Phil.) F.H. Hellw., Adesmia elegans Clos, Ugni molinae Turcz., Ribes punctatum Ruiz et Pav. y Escallonia pulverulenta Pers. (Fig. 2).

El área presenta una superficie inferior a 4 ha con alrededor de 400 individuos adultos y juveniles. En el mes de febrero, solamente algunos se encontraban en floración, y otros en etapa de fructificación, observándose un deterioro de los frutos y semillas por acción del insecto Lithraeus poverus(Blanchard, 1851), Bruchidae. No se observaron indicios de ramoneo por ganado doméstico.

En el sitio los individuos de $A$. bijuga se presentan como arbustos ramosos, con ramas largas, rectas, con la corteza brillante, con ejemplares de cerca de $2 \mathrm{~m}$ de altura, coincidiendo con los antecedentes reportados por Philippi (1884) y Reiche (1897).

Aunque los patrones de regeneración de $A$. bijuga aún se desconocen, observaciones de campo 
Gayana Bot. 65(1), 2008

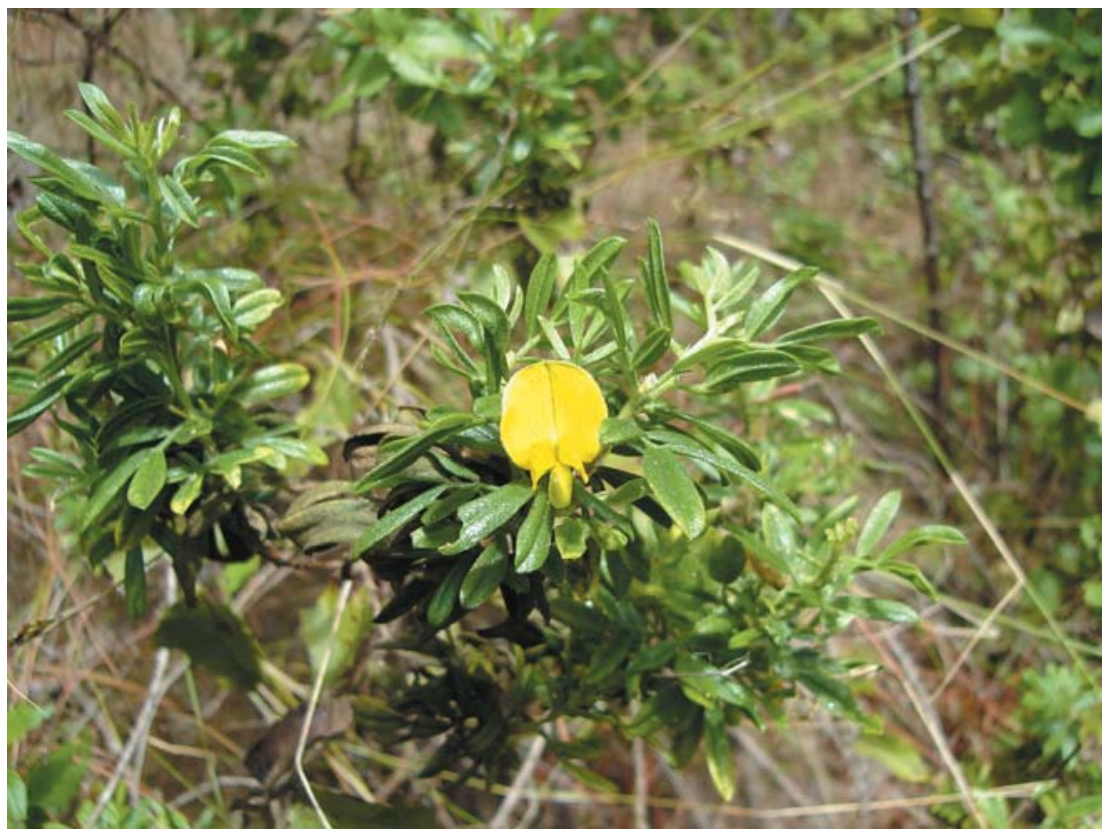

Figura 1. Vista de Adesmia bijuga Phil. en floración (Fotografía de Osvaldo Zuno Delgadillo).

Figure 1. View of Adesmia bijuga Phil. in flowering (Photography by Osvaldo Zuno Delgadillo).

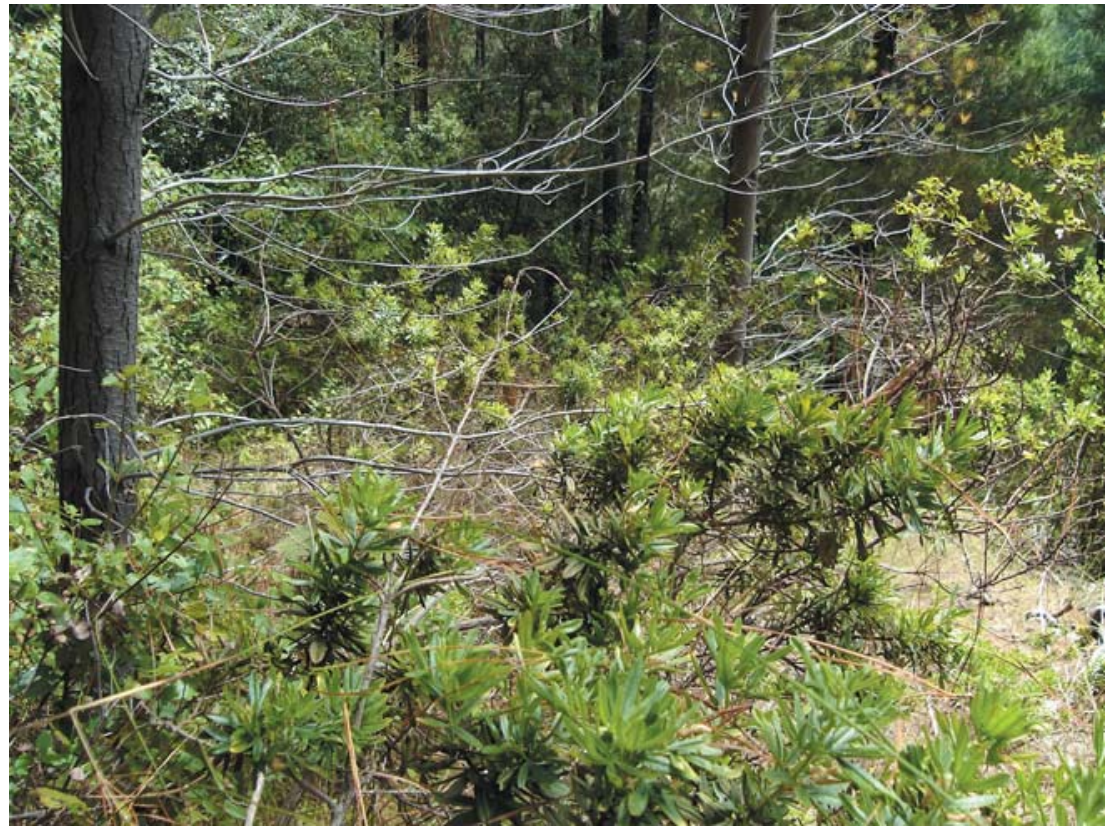

Figura 2. Hábitat de Adesmia bijuga Phil. en una plantación de Pinus radiata y matorral esclerófilo (Fotografía de Osvaldo Zuno Delgadillo).

Figure 2. Habitat of Adesmia bijuga Phil. in a Pinus radiata plantation and sclerophyllous shrub (Photography by Osvaldo Zuno Delgadillo). 
indican que en la especie dominaría la reproducción sexual, evidenciada por una gran producción de plántulas en el sitio. La mayor presencia de plántulas y juveniles se encuentra en el borde entre ambas plantaciones, indicando requerimiento de luminosidad en sus etapas iniciales.

En la actualidad A. bijuga Phil. presenta una distribución restringida, siendo conocida solamente la población aquí descrita. El estado de conservación es desconocido, ya que los escasos registros existentes no dan cuenta de un estudio acabado y sistemático de su distribución.

Material estudiado: Región del Maule. Provincia de Talca, Constitución, Reiche s.n. (Tipo SGO 050435; Clastotipo SI 63344); Constitución, al E de Las Cañas, quebrada los Coigües, $285 \mathrm{~m}$, 35²9'31"'S/72²2'47”'W, 9-XI-2003. S. Hahn s.n. Herbario de la Universidad de Talca, No 2916; Constitución, al E de Las Cañas, quebrada los Coigües, 320 m, 35²8'03”' S/72²4'11’'W, 10-II-2006. P. Gómez \& A. Stoll. Herbario de la Universidad de Talca, $\mathrm{N}^{\circ} 3141$.

\section{AGRADECIMIENTOS}

Se agradece a la Dra. Alexandra Stoll (Universidad de Dresden, Alemania), Dr. José San Martín, Dr. Patricio Peñailillo (IBVB, Universidad de Talca), a Osvaldo Zuno Delgadillo (Universidad de Guadalajara, México), al entomólogo Juan Enrique Barriga (Curicó) y a las Sras. Mélica Muñoz e Inés Mesa, del herbario del Museo Nacional de Historia Natural, Santiago (SGO).

\section{BIBLIOGRAFIA}

Burkart, C. 1967. Contribución al estudio del género Adesmia DC. VII Sinopsis del género Sudamericano de leguminosas Adesmia DC. Darwiniana 14: 463-568.

Marticorena, C. \& M. Quezada. 1985. Catálogo de la flora vascular de Chile. Gayana Botánica 42 (12): 1-157.

Philippi, R. A. 1884. Descripción de algunas plantas nuevas chilenas. Anales de la Universidad de Chile 65: 57-65.

Reiche, C. 1897. Estudios críticos sobre la flora de Chile. Anales de la Universidad de Chile 97: 725-790.

Recibido: 24.09.07

Aceptado:12.12.07 